\title{
Prevalence and risk factors of deep venous thrombosis in patients with longitudinally extensive transverse myelitis: one center data from China
}

Tian Song ${ }^{1,2}$, Xindi Li ${ }^{1,2}$, Yonghong Liü ${ }^{3}$ and Xinghu Zhang ${ }^{1,2^{*}}$

\begin{abstract}
Objective: Deep venous thrombosis (DVT) is a severe complication in longitudinally extensive transverse myelitis (LETM) patients. It may interfere with LETM treatment and delay the recovery of the spinal dysfunction. However, there is less data about the prevalence and risk factors of DVT in patients with LETM. We analyzed data retrospectively to ascertain the prevalence of DVT and the clinical risk factors for DVT.

Methods: Clinical data on 255 LETM patients were collected from medical records. All patients were performed color Doppler ultrasound(US) to screen DVT in both lower extremities when admitted. Clinical characteristics of LETM patients with DVT were compared with those without DVT using corresponding statistical methods. Multivariate logistic regression was performed to identify risk factors related to DVT.

Results: DVT were found in $11.8 \%$ patients with LETM. Univariate analysis showed that age, muscle force and elevated baseline D-dimer were risk factors for DVT. After multivariate logistic regression, age, dyslipidemia, segments of lesions, and elevated baseline D-dimer remained significant independent risk factors.

Conclusions: DVT is common in patients with LETM and related to patient's age, dyslipidemia, segments of lesions, and elevated baseline D-dimer. Early recognition of DVT and thrombosis prophylaxis are appropriate in patients with LETM.
\end{abstract}

Keywords: Deep venous thrombosis, Longitudinally extensive transverse myelitis, Clinical risk factors, Thrombosis prophylaxis

\section{Background}

Longitudinally extensive transverse myelitis (LETM) is defined as myelitis with lesion extending at least 3 continuous vertebral segments in length [1]. A number of conditions can be associated with LETM, including neuromyelitis optica spectrum disorder (NMOSD), spinal infarction, spinal dural arteriovenous fistulas, compressive lesions, metabolic disorders, neoplasm, infection [2]. NMOSD is

\footnotetext{
* Correspondence: xhzhtiantan@hotmail.com

${ }^{1}$ Neuroinfection and Neuroimmunology Center, Department of Neurology, Beijing Tiantan Hospital, Capital Medical University, 6 TiantanXili, Dongcheng District, Beijing 100050, People's Republic of China

${ }^{2}$ China National Clinical Research Center for Neurological Diseases, Beijing Tiantan Hospital, Capital Medical University, 6 TiantanXili, Dongcheng District, Beijing 100050, People's Republic of China

Full list of author information is available at the end of the article
}

the most frequent cause of LETM [2], which primarily attacks the optic nerves and spinal cord [3]. LETM causes complete or partial neurologic impairment of motor, sensory and autonomic system [2]. These conditions are associated with increased morbidity and mortality, and a high economic burden [4]. Venous thromboembolism (VTE) is known as one of the common complications of LETM [5].

VTE includes deep venous thrombosis (DVT) and pulmonary embolism (PE). VTE affects $1 / 1000$ patients, costs $\$ 13.5$ billion annually to treat, and claims 100,000 lives annually in the US [6]. The risk of VTE in individuals with LETM may has been underestimated despite several plausible mechanisms. Autoimmune disorder associated with LETM may upregulate procoagulants,

(c) The Author(s). 2018 Open Access This article is distributed under the terms of the Creative Commons Attribution 4.0 International License (http://creativecommons.org/licenses/by/4.0/), which permits unrestricted use, distribution, and reproduction in any medium, provided you give appropriate credit to the original author(s) and the source, provide a link to the Creative Commons license, and indicate if changes were made. The Creative Commons Public Domain Dedication waiver (http://creativecommons.org/publicdomain/zero/1.0/) applies to the data made available in this article, unless otherwise stated. 
downregulate anticoagulants and suppress fibrinolysis [7].

But so far, little is known about the risk factors for DVT seen in LETM patients as it relates to other conditions, such as postoperation $[8,9]$, cancer $[10,11]$ and connective tissue diseases $[12,13]$. Therefore, we evaluated the prevalence and risk factors of DVT and PE among patients with LETM in this study.

\section{Methods}

We retrospectively collected the medical records of LETM inpatients from July 2011 to August 2014. All patients were performed color Doppler US to screen DVT in both lower extremities when admitted, regardless of symptoms and signs of DVT. US examinations were operated and interpreted by ultrasonic specialists. The deep veins of lower extremities and pelvis were screened at approximately $2-\mathrm{cm}$ intervals in the transverse sections from the inguinal ligament to the ankle. A diagnosis of DVT was confirmed with the presence of visible embolus, dilated incompressible vessel, or abnormal flow pattern [14]. Similarly, D-dimer was tested in all patients when admitted, regardless of symptoms and signs of DVT. When patients complained dyspnea, tachypnea, or chest pain which could not be explained by other reasons, we tested PE using Computed Tomography Pulmonary Angiography (CTPA). Once VTE was detected, anticoagulant was administered.

Continuous variables were described by means \pm SDs, and categorical variables were described as percentages. Characteristics of patients with DVT were compared with those without DVT using corresponding statistical methods. Mann-Whitney U-test was performed for numerical variables (eg, patient's age, inpatient days, inpatient costs, etiology of LETM, length of lesion). Fisher exact probability test was applied for the association of DVT and qualitative variables (eg, sex, combined diseases, location of lesions, contrast-enhanced or not, elevated baseline $\mathrm{D}$-dimer). Ridit analysis was performed for ordinal variables, such as muscle strength of lower limbs. Logistic regression analysis was performed to identify patient characteristics related to DVT. Statistical significance was defined as $P<0.05$. All statistical analysis was performed by IBM SPSS statistical software version 21.0 (IBM, Armonk, NY, USA).

The study protocol was approved by the ethics committee of Beijing Tiantan Hospital. Informed consent was signed by all patients or their legal representatives.

\section{Results}

There were 255 LETM patients enrolled in this study in total. The diagnostic criteria for NMOSD were met in 116 patients (45.5\%). In other 139 patients, an alternative specific diagnosis was made: infectious, 57 ; clinically isolated syndrome (CIS), 47; vascular myelopathy, 9; neoplastic, 5; compressive, 3; nutritional, 3; neurosarcoidosis, 2; and idiopathic, 13. 68 NMOSD cases were at first attack, and 48 cases were recurrent. These patients (186 women, 69 men) ranged in age from 15 to 77 years (mean 41.3 years). DVT was identified in 30 patients, corresponding to $11.8 \%$ of LETM cases, and 4 cases were symptomatic DVT. Symptomatic PE was confirmed in 1 patient by CTPA.

Patients with DVT were at older age, had poorer muscle force of lower limbs and higher baseline D-dimer when compared to those without DVT (Table $1, P<0.001$ ). The associations of lipid disorder, smoking and lesion length with DVT were nearly significant $(P=0.06)$ in univariate analysis. The duration of hospitalization was obviously longer in patients with DVT than that without DVT (31.87 versus 18.95 days, $P<0.001$ ). The hospital charges were higher in patients with DVT than patients absence of DVT (38,178 versus 17,153 CNY, $P<0.001)$. There were no differences in sex, comorbidity, etiology, location and enhancement of lesions, and coagulogram abnormal.

Multivariate logistic regression analysis presented that elderly patients showed higher likelihood of DVT with an OR of 1.10(95\% CI 1.03-1.18). In addition, lipid disorder (OR0.07, 95\%CI 0.01-0.51), lesion segments (OR1.53, 95\%CI 1.14-2.05), elevated baseline D-dimer (OR18.40, 95\%CI 3.32-101.89) were independent risk factors $(P<0.05)$ for occurrence of DVT (Table 2$)$.

\section{Discussion}

This is a retrospective and observational study to identify the prevalence and risk factors of DVT in LETM patients. DVT prevalence among LETM inpatients in our study was $11.8 \%$, obviously higher than that in non-surgical inpatients [11, 15], outpatients [16] and community population [17]. However, previous studies regarding to surgical and spinal cord lesion cases showed inconsistent DVT prevalence, ranging from 1.2\% [18] to $27.6 \%$ [19]. This inconsistence can be explained by difference in population/race, sample size, DVT definition (symptomatic or asymptomatic).

Another primary aim of this study was to analyzed individual-level risk factors of DVT. Our data showed that age, dyslipidemia, lesion segments and elevated baseline D-dimer remained independent risk factors after adjustment for covariates. There are numerous published reports concerning the risk factors for DVT development in various diseases. A study about patients after spine surgery reported that risk factors for DVT included age and preoperative walking disability [20]. Immobility as a risk factor of DVT was also mentioned in neurosurgical patients, including cranial and spinal procedures [9]. However, there were different opinions. A Korean-based study reported that age and 
Table 1 Characteristics of LETM patients with and without DVT

\begin{tabular}{|c|c|c|c|c|}
\hline Variable & $\begin{array}{l}\text { Total } \\
(N=255)\end{array}$ & $\begin{array}{l}\text { With DVT } \\
(N=30)\end{array}$ & $\begin{array}{l}\text { Without DVT } \\
(N=225)\end{array}$ & $P$ value \\
\hline Age (years), mean $\pm S D$ & $41.3 \pm 13.93$ & $53.73 \pm 11.94$ & $39.65 \pm 13.34$ & $<0.001$ \\
\hline \multicolumn{5}{|l|}{ Sex, $n(\%)$} \\
\hline Men & 69 & $7(23.33 \%)$ & $62(27.56 \%)$ & \multirow[t]{2}{*}{0.62} \\
\hline Women & 186 & $23(76.67 \%)$ & $163(72.44 \%)$ & \\
\hline Inpatient stays (days), mean \pm SD & $20.47 \pm 9.90$ & $31.87 \pm 17.68$ & $18.95 \pm 7.14$ & $<0.001$ \\
\hline Inpatient costs (CNY), mean \pm SD & $19,627.22 \pm 14,019.56$ & $38,178.44 \pm 30,309.69$ & $17,153.73 \pm 7190.18$ & $<0.001$ \\
\hline \multicolumn{5}{|l|}{ Comorbidity, $n(\%)$} \\
\hline Hematological abnormality (anemia, leukopenia) & $13(5.10 \%)$ & $1(3.33 \%)$ & $12(5.33 \%)$ & 0.64 \\
\hline Connective tissue diseases & 39(15.29\%) & $5(16.67 \%)$ & $34(15.11 \%)$ & 0.82 \\
\hline Thyroid dysfunction & $22(8.63 \%)$ & $3(10.00 \%)$ & 19(8.44\%) & 0.78 \\
\hline Diabetes mellitus type 2 & $12(4.71 \%)$ & $3(10.00 \%)$ & $9(4.00 \%)$ & 0.14 \\
\hline Hypertension & $27(10.59 \%)$ & $5(16.67 \%)$ & $22(9.78 \%)$ & 0.25 \\
\hline Dyslipidemia & $80(31.37 \%)$ & $5(16.67 \%)$ & 75(33.33\%) & 0.06 \\
\hline Smoking & $42(16.47 \%)$ & $9(30 \%)$ & $33(14.67 \%)$ & 0.06 \\
\hline \multicolumn{5}{|l|}{ Etiology, n(\%) } \\
\hline NMOSD & $116(45.49 \%)$ & 13(43.33\%) & 103(45.78\%) & 0.85 \\
\hline First attack & $68(26.67 \%)$ & $6(20 \%)$ & $62(27.56 \%)$ & \multirow[t]{2}{*}{0.38} \\
\hline Relapses & $48(18.82 \%)$ & $7(23.33 \%)$ & $41(18.22 \%)$ & \\
\hline Infection & $57(22.35 \%)$ & $9(30 \%)$ & $48(21.33 \%)$ & 0.35 \\
\hline $\mathrm{CIS}$ & $47(18.43 \%)$ & $4(13.33 \%)$ & $43(19.11 \%)$ & 0.62 \\
\hline Vascular myelopathy & $9(3.53 \%)$ & $1(3.33 \%)$ & $8(3.56 \%)$ & 0.71 \\
\hline Neoplastic & $5(1.96 \%)$ & $1(3.33 \%)$ & $4(1.78 \%)$ & 0.47 \\
\hline Compressive & $3(1.18 \%)$ & 0 & $3(1.33 \%)$ & 1.00 \\
\hline Nutritional & $3(1.18 \%)$ & 0 & $3(1.33 \%)$ & 1.00 \\
\hline Neurosarcoidosis & $2(0.78 \%)$ & 0 & $2(0.89 \%)$ & 1.00 \\
\hline Idiopathic & $13(5.10 \%)$ & $2(6.67 \%)$ & $11(4.89 \%)$ & 0.66 \\
\hline \multicolumn{5}{|l|}{ Muscle strength of left leg, $n(\%)$} \\
\hline Grade 0 & 19(7.45\%) & $8(26.67 \%)$ & $11(4.89 \%)$ & \multirow[t]{6}{*}{$<0.001$} \\
\hline Grade 1 & $7(2.75 \%)$ & $2(6.67 \%)$ & $5(2.22 \%)$ & \\
\hline Grade 2 & $14(5.49 \%)$ & $5(16.67 \%)$ & $9(4.00 \%)$ & \\
\hline Grade 3 & $25(9.80 \%)$ & $4(13.33 \%)$ & $21(9.33 \%)$ & \\
\hline Grade 4 & $73(28.63 \%)$ & $5(16.67 \%)$ & $68(30.22 \%)$ & \\
\hline Grade 5 & $117(45.88 \%)$ & $6(20.00 \%)$ & $111(49.33 \%)$ & \\
\hline \multicolumn{5}{|l|}{ Muscle strength of right leg, $n(\%)$} \\
\hline Grade 0 & $19(7.45 \%)$ & $8(26.67 \%)$ & $11(4.89 \%)$ & \multirow[t]{6}{*}{$<0.001$} \\
\hline Grade 1 & 10(3.92\%) & $4(13.33 \%)$ & $6(2.67 \%)$ & \\
\hline Grade 2 & $11(4.31 \%)$ & $4(13.33 \%)$ & $7(3.11 \%)$ & \\
\hline Grade 3 & $21(8.24 \%)$ & $4(13.33 \%)$ & $17(7.56 \%)$ & \\
\hline Grade 4 & $68(26.67 \%)$ & $8(26.67 \%)$ & $60(26.67 \%)$ & \\
\hline Grade 5 & $126(49.41 \%)$ & $2(6.67 \%)$ & $124(55.11 \%)$ & \\
\hline \multicolumn{5}{|l|}{ Location of lesions, $n$ (\%) } \\
\hline Cervical & 204(80.00\%) & $25(83.33 \%)$ & $179(79.56 \%)$ & 0.63 \\
\hline Thoracic & $133(52.16 \%)$ & $17(56.67 \%)$ & $116(51.56 \%)$ & 0.60 \\
\hline
\end{tabular}


Table 1 Characteristics of LETM patients with and without DVT (Continued)

\begin{tabular}{|c|c|c|c|c|}
\hline Variable & $\begin{array}{l}\text { Total } \\
(N=255)\end{array}$ & $\begin{array}{l}\text { With DVT } \\
(N=30)\end{array}$ & $\begin{array}{l}\text { Without DVT } \\
(N=225)\end{array}$ & $P$ value \\
\hline Lumbar & $8(3.14 \%)$ & 1(3.33\%) & $7(3.11 \%)$ & 1.00 \\
\hline Segments of lesions, mean \pm SD & $5.20 \pm 3.56$ & $6.77 \pm 4.98$ & $5 \pm 3.30$ & 0.06 \\
\hline \multicolumn{5}{|l|}{ Enhancement of lesions, $n$ (\%) } \\
\hline Yes & $115(45.10 \%)$ & $13(43.33 \%)$ & $102(45.33 \%)$ & \multirow[t]{3}{*}{0.60} \\
\hline No & $108(42.35 \%)$ & $11(36.67 \%)$ & $97(43.11 \%)$ & \\
\hline Unknown & $32(12.55 \%)$ & $6(20.00 \%)$ & $26(11.56 \%)$ & \\
\hline $\begin{array}{l}\text { Internals between LETM symptoms onset and US screening(days), } \\
\text { mean } \pm \text { SD }\end{array}$ & $18.45 \pm 7.40$ & $21.40 \pm 10.26$ & $18.07 \pm 6.87$ & 0.025 \\
\hline Coagulogram abnormal, n (\%) & $108(42.35 \%)$ & $14(46.67 \%)$ & $94(41.78 \%)$ & 0.61 \\
\hline Baseline D-dimer elevated, n (\%) & $24(9.41 \%)$ & $17(56.67 \%)$ & $7(3.11 \%)$ & $<0.001$ \\
\hline
\end{tabular}

completeness of motor paralysis were not significantly associated with occurrence of DVT after spinal cord injury [21]. In our study, immobility appeared to be a risk factor in univariate analysis, but it was no longer independent risk factor after multivariate analysis. D-dimer values, in particular age-adjusted D-dimer cut-off values, have been confirmed to be associated with DVT [22, 23]. Our finding is in accordance with above researches. A review showed lipoprotein (a) was slightly but significantly associated with an increased risk of VTE (OR: 1.56, 95\% CI: 1.36, 1.79), which included 10 studies, 13,541 patients [24]. Our study analyzed dyslipidemia as a whole including hyper/hypo-triglyceridemia and hyper/hypo- hypercholesteremia, instead of lipoprotein (a) individually. Much of the differences between studies may be due to small patient populations and different methods for assessment of DVT [25]. We have not searched literature focused on risk factors of DVT in LETM patients. Therefore,larger prospective studies will be needed to illuminate the risk factors for DVT and intrinsic mechanism.

The plausible mechanisms to explain the high prevalence of DVT in LETM patients remain unclear. Three main promoters to venous thrombosis included: venous stasis, hypercoagulable blood state and vascular endothelial injury (Virchow's triad) [26]. Immobility caused by

Table 2 Multivariate logistic regression analysis of significant risk factors for DVT in LETM patients

\begin{tabular}{lll}
\hline & $P$ & $\mathrm{OR}(95 \% \mathrm{Cl})$ \\
\hline Age & 0.004 & $1.104(1.032-1.181)$ \\
Dyslipidemia & 0.009 & $0.07(0.01-0.51)$ \\
Segments of lesions & 0.005 & $1.529(1.140-2.050)$ \\
Baseline D-dimer elevated & $<0.001$ & $18.395(3.321-101.891)$ \\
\hline
\end{tabular}

spinal cord dysfunction can lead to venous stasis in LETM cases. There is some evidence that steroid use can increase procoagulant factors [27]. Most NMOSD and CIS patients in our study received high dose steroid pulse therapy. In addition, hospitalization also is a known risk factor for VTE [28]. Above elements may contribute to the high prevalence of DVT in LETM inpatients.

Several limitations to our analysis are worth noting. First limitation is lack of prospective control, which is inherent to observational studies. Second, the interval was not standardized between LETM symptom onset and US screening. The intervals in patients with DVT were significantly longer $(P<0.05$, Table 1$)$. The prevalence in our study might be underestimated because US screening was performed beyond the acute phase in some cases. Third, certain confounders were unavailable in our databases, such as body mass index(BMI) [29] and glucocorticoid usage [30], which are known as risk factors for DVT. Despite these limitations, our data is convincing for the large sample size and routine US screening for DVT.

\section{Conclusion}

In conclusion, this retrospective study demonstrated that patients with LETM had an increased risk for developing DVT. Therefore, it is important to identify the high-risk patients and initiate thrombosis prophylaxis promptly.

\footnotetext{
Abbreviations

BMI: body mass index; CIS: Clinical isolated syndrome; CNS: Central nervous system; CTPA: Computed Tomography Pulmonary Angiography; DVT: Deep venous thrombosis; LETM: Longitudinally extensive transverse myelitis; NMOSD: Neuromyelitis optica spectrum disorders; PE: Pulmonary embolism; US: Ultrasound; VTE: Venous thromboembolism
} 


\section{Acknowledgements}

The authors thank the participants and all who were involved in this study.

\section{Funding}

This work was supported by Youth Research Fund of Beijing Tiantan Hospital (201403011)

\section{Availability of data and materials}

All necessary data generated or analyzed during this study are included in this published article (results section, tables). Individual patient data that support the findings of this analysis are available upon request from the corresponding author on reasonable request.

\section{Authors' contributions}

ST contributed to study design, data analysis, manuscript writing and revising. LXD contributed to patient's clinical information collection and follow-up. LYH contributed to patient's clinical information collection and follow-up. ZXH contributed to study design and manuscript revising. All authors read and approved the final manuscript.

\section{Ethics approval and consent to participate}

Written informed consent was obtained from all patients or their legal representatives, and this study was approved by the Ethics Committee of Beijing Tiantan Hospital.

\section{Consent for publication}

Not applicable.

\section{Competing interests}

The authors declare that they have no competing interests.

\section{Publisher's Note}

Springer Nature remains neutral with regard to jurisdictional claims in published maps and institutional affiliations.

\section{Author details}

'Neuroinfection and Neuroimmunology Center, Department of Neurology, Beijing Tiantan Hospital, Capital Medical University, 6 TiantanXili, Dongcheng District, Beijing 100050, People's Republic of China. ${ }^{2}$ China National Clinical Research Center for Neurological Diseases, Beijing Tiantan Hospital, Capital Medical University, 6 TiantanXili, Dongcheng District, Beijing 100050, People's Republic of China. ${ }^{3}$ Department of Neurology, Beijing Rehabilitation Hospital, Capital Medical University,Xixiazhuang, Shijingshan District, Beijing 100050, People's Republic of China.

Received: 17 August 2017 Accepted: 16 October 2018

Published online: 30 October 2018

\section{References}

1. Weinshenker BG, Wingerchuk DM, Vukusic S, Linbo L, Pittock SJ, Lucchinetti CF, et al. Neuromyelitis optica lgG predicts relapse after longitudinally extensive transverse myelitis. Ann Neurol. 2006;59:566-9.

2. Tobin WO, Weinshenker BG, Lucchinetti CF. Longitudinally extensive transverse myelitis. Curr Opin Neurol. 2014;27:279-89.

3. Wingerchuk DM, Banwell B, Bennett UL, Cabre P, Carroll W, Chitnis T, et al. International consensus diagnostic criteria for neuromyelitis optica spectrum disorders. Neurology. 2015;85:177-89.

4. ZhangBao J, Zhou L, Li X, Cai T, Lu J, Lu C, et al. The clinical characteristics of AQP4 antibody positive NMO/SD in a large cohort of Chinese Han patients. J Neuroimmunol. 2017;302:49-55.

5. Cushman M. Epidemiology and risk factors for venous thrombosis. Semin Hematol. 2007:44:62-9.

6. Behravesh S, Hoang P, Nanda A, Wallace A, Sheth RA, Deipolyi AR, et al. Pathogenesis of thromboembolism and endovascular management. Thrombosis. 2017;2017, 3039713.

7. Xu J, Lupu F, Esmon CT. Inflammation, innate immunity and blood coagulation. Hamostaseologie. 2010;30:5-6, 8-9.

8. Sebastian AS, Currier BL, Kakar S, Nguyen EC, Wagie AE, Habermann ES, et al. Risk factors for venous thromboembolism following thoracolumbar surgery: analysis of 43,777 patients from the American
College of Surgeons National Surgical Quality Improvement Program 2005 to 2012. Global Spine J. 2016;6:738-43.

9. Rolston JD, Han SJ, Bloch O, Parsa AT. What clinical factors predict the incidence of deep venous thrombosis and pulmonary embolism in neurosurgical patients? J Neurosurg. 2014;121:908-18.

10. Faiz AS, Khan I, Beckman MG, Bockenstedt P, Heit JA, Kulkarni R, et al. Characteristics and risk factors of Cancer associated venous thromboembolism. Thromb Res. 2015;136:535-41.

11. Mellema WW, van der Hoek D, Postmus PE, Smit EF. Retrospective evaluation of thromboembolic events in patients with non-small cell lung cancer treated with platinum-based chemotherapy. Lung Cancer. 2014;86:73-7.

12. Lee JJ, Pope JE. A meta-analysis of the risk of venous thromboembolism in inflammatory rheumatic diseases. Arthritis Res Ther. 2014;16:435

13. Kim SC, Schneeweiss S, Liu J, Solomon DH. Risk of venous thromboembolism in patients with rheumatoid arthritis. Arthritis Care Res (Hoboken). 2013;65:1600-7.

14. Liu LP, Zheng HG, Wang DZ, Wang YL, Hussain M, Sun HX, et al. Risk assessment of deep-vein thrombosis after acute stroke: a prospective study using clinical factors. CNS Neurosci Ther. 2014;20:403-10.

15. Lawall H, Hoffmanns W, Hoffmanns P, Rapp U, Ames M, Pira A, et al. Prevalence of deep vein thrombosis (DVT) in non-surgical patients at hospital admission. Thromb Haemost. 2007;98:765-70.

16. Shulman RM, Buchan C, Bleakney RR, White LM. Low prevalence of unexpected popliteal DVT detected on routine MRI assessment of the knee. Clin Imaging. 2016:40:79-85.

17. Ho WK, Hankey GJ, Eikelboom JW. The incidence of venous thromboembolism: a prospective, community-based study in Perth. Western Australia Med J Aust. 2008;189:144-7.

18. Maiser S, Adil MM, Roohani P, Tariq N. Patients with transverse myelitis who developed venous thromboembolism while hospitalized have increased rate for inpatient mortality. J Neuroimmunol. 2013;261:120-2.

19. Do JG, Kim DH, Sung DH. Incidence of deep vein thrombosis after spinal cord injury in Korean patients at acute rehabilitation unit. J Korean Med Sci. 2013;28:1382-7.

20. Tominaga H, Setoguchi T, Tanabe F, Kawamura I, Tsuneyoshi $Y$, Kawabata $\mathrm{N}$, et al. Risk factors for venous thromboembolism after spine surgery. Medicine. 2015;94:e466.

21. Do JG, Kim DH, Sung DH. Incidence of deep vein thrombosis after spinal cord injury in Korean patients at acute rehabilitation unit. J Korean Med Sci. 2013;28:1382

22. Haase C, Joergensen M, Ellervik C, Joergensen MK, Bathum L. Age- and sex-dependent reference intervals for D-dimer: evidence for a marked increase by age. Thromb Res. 2013;132:676-80.

23. Broen K, Scholtes B, Vossen R. Predicting the need for further thrombosis diagnostics in suspected DVT is increased by using age adjusted D-dimer values. Thromb Res. 2016;145:107-8.

24. Dentali F, Gessi V, Marcucci R, Gianni M, Grandi AM, Franchini M. Lipoprotein(a) as a risk factor for venous thromboembolism: a systematic review and meta-analysis of the literature. Semin Thromb Hemost. 2017.

25. Guyatt GH, Eikelboom JW, Gould MK, Garcia DA, Crowther M, Murad MH, et al. (2012) Approach to outcome measurement in the prevention of thrombosis in surgical and medical patients: antithrombotic therapy and prevention of thrombosis, 9th ed: American College of Chest Physicians Evidence-Based Clinical Practice Guidelines. Chest 141: e185S-e194S.

26. Kumar DR, Hanlin E, Glurich I, Mazza JJ, Yale SH. Virchow's contribution to the understanding of thrombosis and cellular biology. Clin Med Res. 2010;8:168-72.

27. Girolami A, de Marinis GB, Bonamigo E, Treleani M, Vettore S. Arterial and venous thromboses in patients with idiopathic (immunological) thrombocytopenia: a possible contributing role of cortisone-induced hypercoagulable state. Clin Appl Thromb Hemost. 2013;19:613-8.

28. Heit JA, Melton LR, Lohse CM, Petterson TM, Silverstein MD, Mohr DN, et al. Incidence of venous thromboembolism in hospitalized patients vs community residents. Mayo Clin Proc. 2001;76:1102-10.

29. Holst $A G$, Jensen $G$, Prescott E. Risk factors for venous thromboembolism: results from the Copenhagen City heart study. Circulation. 2010;121:1896-903.

30. Johannesdottir SA, Horvath-Puho E, Dekkers OM, Cannegieter SC, Jorgensen JO, Ehrenstein $\mathrm{V}$, et al. Use of glucocorticoids and risk of venous thromboembolism: a nationwide population-based case-control study. JAMA Intern Med. 2013;173:743-52. 